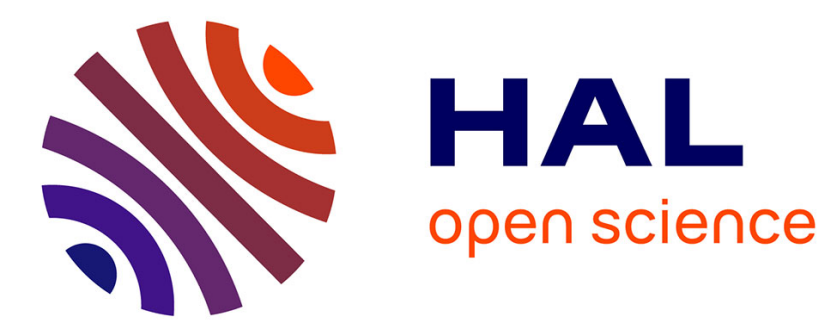

\title{
Perspectives of the study of double Pomeron exchange at the LHC
}

\author{
J.P. Guillaud, A.E. Sobol
}

\section{To cite this version:}

J.P. Guillaud, A.E. Sobol. Perspectives of the study of double Pomeron exchange at the LHC. Lomonosov Conference on Elementary Particle Physics 11, Aug 2003, Moscow, Russia. pp.1-16. in2p3-00021763

\section{HAL Id: in2p3-00021763 https://hal.in2p3.fr/in2p3-00021763}

Submitted on 10 May 2004

HAL is a multi-disciplinary open access archive for the deposit and dissemination of scientific research documents, whether they are published or not. The documents may come from teaching and research institutions in France or abroad, or from public or private research centers.
L'archive ouverte pluridisciplinaire HAL, est destinée au dépôt et à la diffusion de documents scientifiques de niveau recherche, publiés ou non, émanant des établissements d'enseignement et de recherche français ou étrangers, des laboratoires publics ou privés. 
LAPP-EXP 2003-09

September 2003

\title{
Perspectives of the study of double Pomeron exchange at the $\mathrm{LHC}$
}

\author{
J.P. Guillaud, A.Sobol \\ LAPP, IN2P3-CNRS, Chemin de Bellevue, BP110, \\ F-74941, Annecy-le-Vieux
}

\begin{abstract}
We discuss the theoretical estimates and perspectives of the measurements of doubly diffractive production in central proton-proton collisions, $p p \rightarrow p X p$ at LHC energy, where a pure double Pomeron exchange is predicted. Such study can be made by measurements using one of the two general-purpose LHC detectors, CMS or ATLAS, and with a set of special tracking detectors located close to the beam at distances more than 100 meters from the interaction point to detect the diffractively scattered protons. This study is interesting due to the intensive glueball production expected in the mass region below $10 \mathrm{GeV}$ and due to the unique possibility of the measurements of exclusive Higgs production in double Pomeron exchange.
\end{abstract}

Talk given at the 11th Lomonosov Conference on Elementary Particle Physics , 21-27 august 2003, Moscow, Russia 


\section{Introduction}

This paper is mainly devoted to the exclusive measurements of central production in proton-proton collisions:

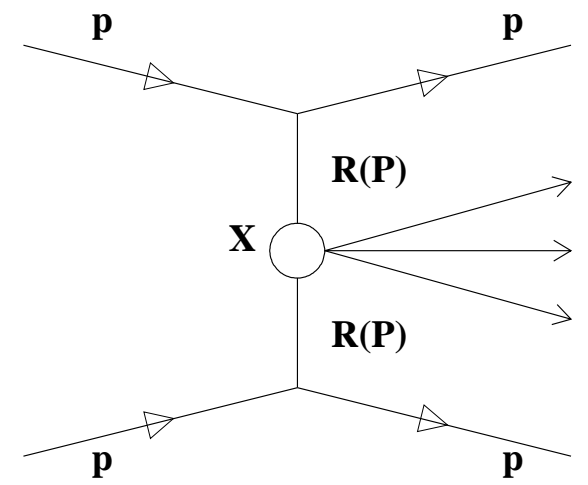

$$
p p \rightarrow p X p
$$

Figure 1: Diagram of the central production in proton-proton collisions.

The $X$, produced in the interaction of the two exchanged particles which can be Reggeons $(R)$ or Pomerons $(P)$, is called a central particle (or system) due to its pseudorapidity which is close to 0 . Such reactions are predicted to be a source of glueballs or gluonic rich states when both of the exchanged particles are Pomerons (Double Pomeron Exchange (DPE)).

The reaction 1 has been studied at CERN in fixed target experiments at different energy, WA76 $(\sqrt{s}=12.7$ and $23.8 \mathrm{GeV})$, WA91, NA12/2, WA102 $(\sqrt{s}=29.1 \mathrm{GeV})$, as well at colliders, UA8 at CERN $\operatorname{Sp} \bar{p} \mathrm{~S}(\sqrt{s}=630 \mathrm{GeV})$. WA102 experiment produced the largest and detailed data set at low energy. Many new results have been obtained in hadron spectroscopy (see, for example, [1]), in particular, efforts have been made to find new kinematic variables which could separate states with a strong gluon component from ordinary mesons. Two interesting effects are observed by the Collaboration.

One of them is the so-called glueball-filter. It was proposed to analyse the data at different values of the kinematic variable $d P_{T}$, the difference between the transverse momentum vectors of the exchanged particles ${ }^{1}$. The Collaboration obtained the ratio, $R$, of the production cross-section for small $d P_{T}(\leq 0.2 \mathrm{GeV})$ and large $d P_{T}(\geq 0.5 \mathrm{GeV})$ for different resonances [1]. It was observed that the resonances studied can be separated into 3 groups according to $R$. The values of $R$ for different resonances are shown in figure 2. It is interesting to note (left part of the figure) that all the undisputed $q \bar{q}$ states, namely those with positive $G$ parity and $I=0$, have a very small value for this ratio $(\leq 0.1)$. Some of the states (central part) with $I=1$ or negative $G$ parity, which cannot be produced by double Pomeron exchange, have a slightly higher value $(\approx 0.25)$. All the

\footnotetext{
${ }^{1} d P_{T}=\sqrt{\left(P_{1}^{x}-P_{2}^{x}\right)^{2}+\left(P_{1}^{y}-P_{2}^{y}\right)^{2}}$, where $P_{1}$ and $P_{2}$ are the momenta of the exchanged particles.
} 
states which can be considered as candidates for glueballs (right part) have a large value for this ratio, close to 1 . Until now, this effect has not had any convincing theoretical explanation, but it can indicate the possibility of a glueball- $q \bar{q}$ filter mechanism in central production. With such a filter, all the undisputed $q \bar{q}$ states are observed to be suppressed at small $d P_{T}$, but the glueball candidates $f_{0}(1500), f_{0}(1710), f_{2}(1950)$, together with the enigmatic $f_{0}(980)$, survive.

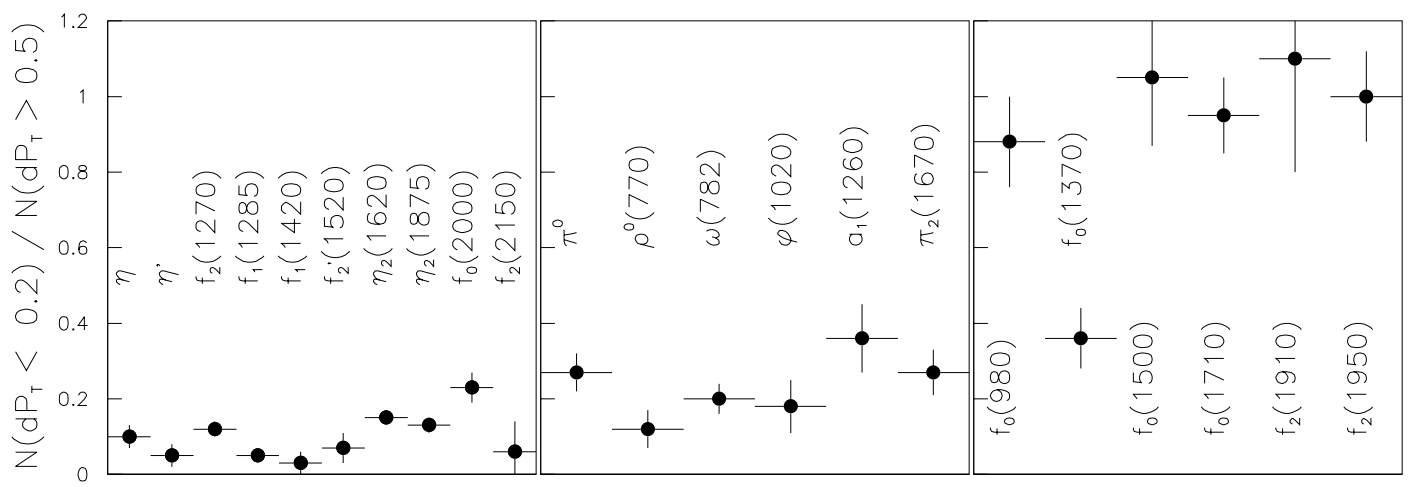

Figure 2: The ratio, $R$, of the production cross-section for small $d P_{T}(\leq 0.2 \mathrm{GeV})$ and large $d P_{T}(\geq 0.5 \mathrm{GeV})$ for different resonances.

The second effect observed in the WA102 experiment is an interesting behaviour of the azimuthal angle, $\phi_{p p}$, between the transverse momentum vectors of the outgoing protons. Naively, it would have been expected that this angle should be flat irrespective of the resonances produced. The experimentally observed $\phi_{p p}$ dependences are clearly non-flat and considerable variations are found between resonances with different $J^{P C}$. Figure 3 shows the $\phi_{p p}$-dependences for several resonances. Several theoretical papers have been published on the $\phi_{p p}$-dependence $[2,3]$. All agree that the exchanged particle must have $J>0$ and that $J=1$ is the simplest explanation. Using $\gamma \gamma$ collisions as an analogy, Close and Schuler [3] have calculated the $\phi_{p p}$ and $t^{2}$ dependences for the production of resonances with different $J^{P C}$. In their model of double Pomeron exchange the Pomeron acts as a nonconserved vector current. In the reference [4] this model was tested for some resonances with $J^{P C}=0^{-+}, 1^{++}, 2^{-+}, 0^{++}, 2^{++}$. For these states, a good description of the experimental data was obtained. It is interesting that $\phi_{p p}$ has a different behaviour for the undisputed $q \bar{q}$ states and for the glueball candidates with identical quantum numbers $J^{P C}\left(f_{0}(1370)\right.$ and $f_{0}(1500)$ with $J^{P C}=0^{++}, f_{2}(1270)$ and $f_{2}(1950)$ with $\left.J^{P C}=2^{++}\right)$.

Attempts to find some kind of a filter for the separation of gluon rich states from ordinary ones are very important because the question "where is a glueball ?" is still open. Some authors suggest scenarios in which a scalar glueball mixes with two $q \bar{q}$ states. F.Close and A.Kirk [5] on the basis of the WA102 data predict the following mixing

\footnotetext{
${ }^{2} t$ is the transverse momentum squared between the incoming and outgoing protons.
} 


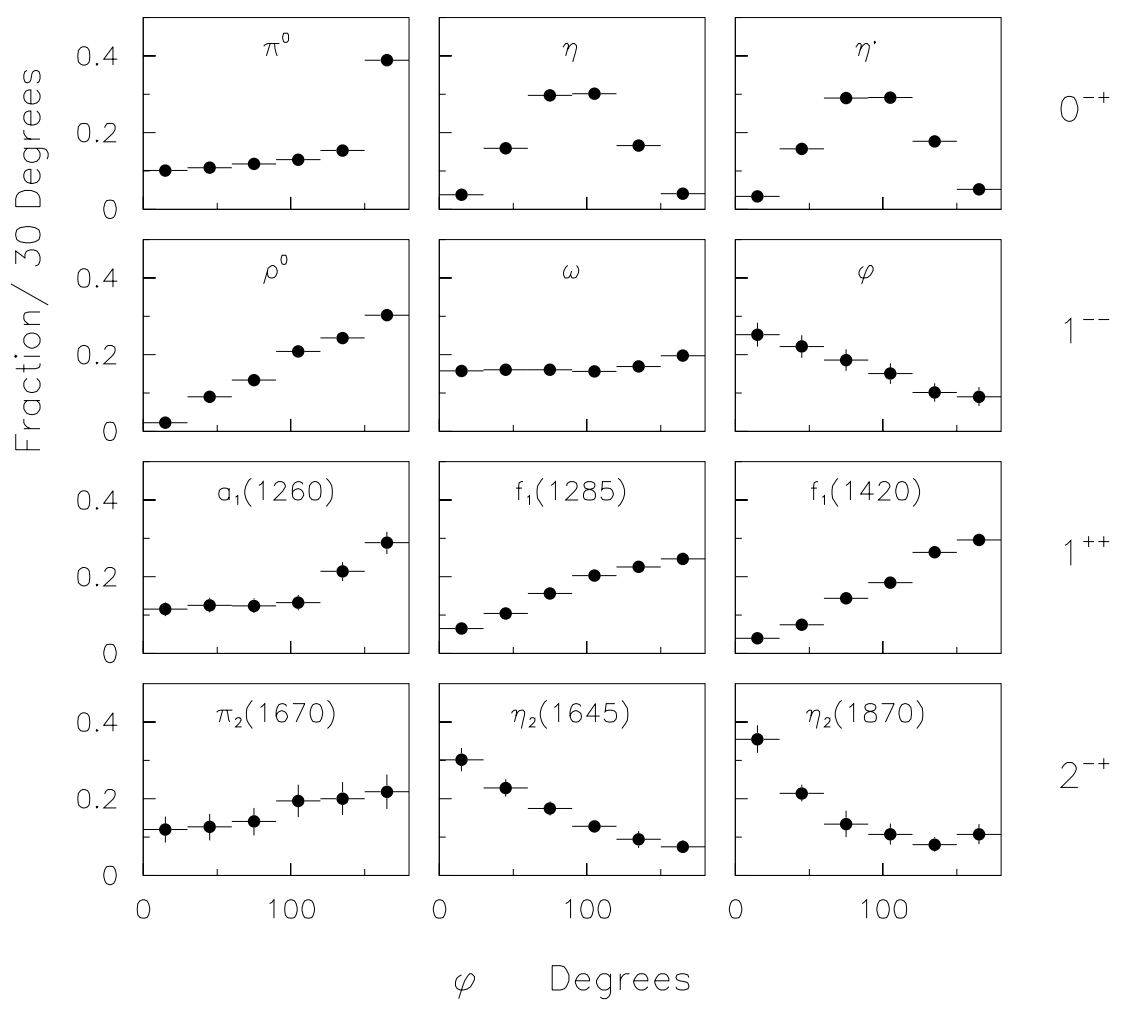

Figure 3: Azimuthal angle $\phi_{p p}$ between the transverse momentum vectors for the outgoing protons for resonances with $J^{P C}=0^{-+}, 1^{--}, 1^{++}$and $2^{-+}$.

scheme for $f_{0}(1370), f_{0}(1500)$ and $f_{0}(1750)$ scalars:

$$
\begin{aligned}
& f_{0}(1370)=-0.79 \frac{1}{\sqrt{2}}(u \bar{u}+d \bar{d})-0.13 s \bar{s}+0.60 \text { glueball } \\
& f_{0}(1500)=-0.62 \frac{1}{\sqrt{2}}(u \bar{u}+d \bar{d})+0.37 s \bar{s}-0.69 \text { glueball } \\
& f_{0}(1750)=0.14 \frac{1}{\sqrt{2}}(u \bar{u}+d \bar{d})+0.91 s \bar{s}+0.39 \text { glueball }
\end{aligned}
$$

Now several different receipts of the mixing are suggested by other authors [6]. Unfortunately they give absolutely different values for the quarkonium and glueball contributions to these 3 scalar states ${ }^{3}$.

However, there is some optimism to solve the "glueball puzzle" at higher energy based on some theoretical estimates and on the recent result of the UA8 Collaboration [8] at CERN. Indeed there are theoretical predictions [9] for the intensities of the different types of exchange in function of the centre of mass energy:

$$
\begin{aligned}
\sigma(R R) & \sim s^{-1}, \\
\sigma(R P) & \sim s^{-0.5}, \\
\sigma(P P) & \sim \text { constant },
\end{aligned}
$$

\footnotetext{
${ }^{3}$ All these mixing scenarios are collected and discussed in the review of E.Klempt [7]
} 
where $R R, R P$ and $P P$ mean respectively Reggeon-Reggeon, Reggeon-Pomeron and Pomeron-Pomeron exchanges. These show that the contribution of the double Pomeron exchange in relation to the Reggeon-Reggeon and Pomeron-Pomeron exchanges in the cross-section of reaction 1 increases with the increase of the energy. So the production of central resonances with a rich gluon component becomes dominant. The results obtained by the experiments WA76, WA91 and WA102 [10] at different energies confirm the theoretical predictions 2 shown above. For example, the production of $\rho^{\circ}(770)$, which has an isospin 1 and cannot be produced in a DPE, becomes less important with increasing $s$. The $\eta^{\prime}(958)$ production, that experimentally does not depend on $s$, can be explained naturally by a double Pomeron exchange.

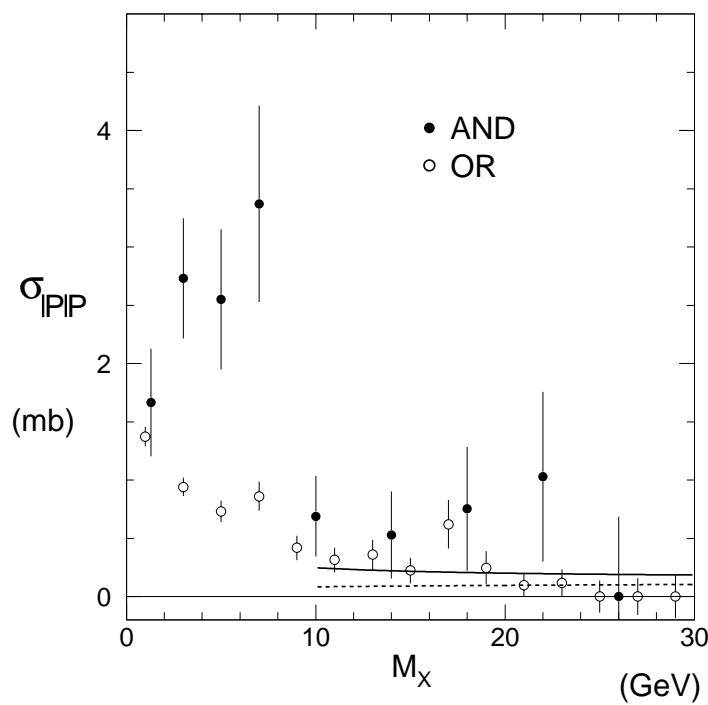

Figure 4: Mass dependence for $X$ production from the "AND" and "OR" data samples $^{4}$ of UA8 experiment at CERN $S p \bar{p} S$ at $\sqrt{s}=630 \mathrm{GeV}$.

Recently UA8 Collaboration [8] reported new results based on its 1989 year data sample at the CERN $S p \bar{p} S$ collider. In particular, they studied the reaction $p \bar{p} \rightarrow p X \bar{p}$ at $\sqrt{s}=630 \mathrm{GeV}$, where $X$ is a set of hadrons in the central rapidity region. The dependence of the total cross-section as function of the $X$ mass is shown on the figure by black circles. The white circles show the cross-section for the $X$ production in the single diffraction: $p \bar{p} \rightarrow p X$ or $p \bar{p} \rightarrow X \bar{p}^{4}$. The data display a wide maximum in the mass region up to $10 \mathrm{GeV}$ with a cross-section, few mb, that is an order of magnitude larger than the one predicted. This could be explained by an intensive resonance glueball production in this region of mass. Unfortunately, the UA8 detector was a calorimeter with a mass resolution about $2 \mathrm{GeV}$, too poor to see any mass structure in the cross-section.

At this point, we can answer to the question: "Why would it be of great interest to extend the study of the central production reaction at such a high LHC energy?" In the WA102 experiment at $\sqrt{s} \approx 30 \mathrm{GeV}$ we had approximately equal contributions

\footnotetext{
${ }^{4}$ Black points are called "AND" because both nucleons ( $p$ and $\bar{p}$ ) were detected. In the data pointed by white circles and called "OR" only one nucleon was detected.
} 
of Reggeon-Reggeon $(R R)+$ Reggeon-Pomeron $(R P)$ and Pomeron-Pomeron $(P P)$ exchanges: $\left(\sigma_{R R}+\sigma_{R P}\right) / \sigma_{P P} \approx 1$. In UA8 at $\sqrt{s}=630 \mathrm{GeV}$ this ratio is $\approx 0.05 \mathrm{GeV}$ and at the LHC energy, $\sqrt{s}=14 \mathrm{TeV}$, it would be $\approx 0.001$. So at LHC energies we can expect a pure Double Pomeron Exchange and an absence of Reggeon exchanges of any kind. This creates a favourable condition for gluon rich states production (LHC can be considered as a "glueball factory") and a comfortable backgroundless condition for their observation comparing with a lower energy. The UA8 result looks very encouraging and allows the expectation of a more exciting result at the LHC energy. Of course, the mass resolution should noticeably be improved compared to UA8 to study possible structures in the low mass region.

The cross-section for DPE at the LHC energy can be estimated using the results of WA102 $\left(\sigma_{D P E}=0.14 \mathrm{mb}\right.$ at $\left.\sqrt{s}=28 \mathrm{GeV}\right)$ and equations 2 . From these one can see that $\sigma_{D P E}$ is approximately constant in terms of $s$. To be more precise, theory predicts that $\sigma_{D P E}$ depends on $s$ as $s^{0.08}[11]$. Thus, we can assume that at $\sqrt{s}=14 \mathrm{TeV} \sigma_{D P E}=0.37 \mathrm{mb}$ giving $\approx 350000 \mathrm{DPE}$ events/day (non-corrected by any efficiency) at a luminosity as low as $L=10^{28} \mathrm{~cm}^{-2} \mathrm{~s}^{-1}$ (it will be explained below why we use this value for the luminosity).

The advantages of a study of the central production (reaction 1) at the LHC energy are more striking if one shifts the study of low $X$ mass (several $\mathrm{GeV}$ ) to much heavier objects. One of the main goals of the LHC is the search for the Higgs boson. It turns out, that the Higgs can be produced in the central proton-proton collisions (reaction 1) by DPE exchange. Some estimates of the cross-section for the exclusive central Higgs production at LHC energy are listed in the table below:

\begin{tabular}{|c|c|c|c|}
\hline Year & Publication & $\mathrm{M}_{H}, \mathrm{GeV}$ & $\sigma_{H}, \mathrm{fb}$ \\
\hline 1991 & {$[12]$} & {$[100,400]$} & $100 \div 200$ \\
\hline 1995 & {$[13]$} & {$[100,400]$} & $1000 \div 2000$ \\
\hline 2000 & {$[14]$} & 100 & $10 \div 270$ \\
\hline 2001 & {$[15]$} & {$[100,400]$} & $80 \div 140$ \\
\hline 2002 & {$[16]$} & 120 & $\approx 3$ \\
\hline 2003 & {$[17]$} & {$[100,500]$} & $0.2 \div 8.5$ \\
\hline
\end{tabular}

Table 1: Theoretical predictions for Higgs production in DPE at $\sqrt{s}=14 \mathrm{TeV}$.

If we consider a cross-section $\sigma_{H}=100 \mathrm{fb}$ as an optimistic estimate given by the table 1 one will get more then 30000 exclusive Higgs/year produced by DPE at the nominal LHC luminosity $L=10^{34} \mathrm{~cm}^{-2} \mathrm{~s}^{-1}$. Exclusive measurements allow well to suppress the signal/background ratio $(S / B)$. As shown in reference [18] the significance $S / \sqrt{S+B}$ of the Higgs observation in the process $p p \rightarrow p H p, H \rightarrow b \bar{b}$ during one year at a luminosity $10^{33}$ is about $3 \sigma$ even for the more pessimistic estimate for the Higgs cross-section of 3 $\mathrm{fb}(!)$. This value should be checked in a detailed full-GEANT simulation, but, if it is 
true, then the exclusive Higgs production by DPE would become competitive to the other Higgs channel, like $H \rightarrow \gamma \gamma$ for which $S / \sqrt{S+B}$ is about $4 \sigma$.

In addition to glueballs and Higgs as central systems in the reaction 1, other objects (like 2 jets, light or heavy quarkonium, exotic extra dimension particle) can be studied.

\section{Perspectives of the measurements}

\subsection{Low luminosity physics}

To study an exclusive central production (reaction 1) we should measure the central particle $X$ or its decay products and the 2 protons of the final state of the reaction. The typical pseudorapidity distributions are shown in fig. 5 for the process, where $X$ decays to $2 \pi^{0}$ with $4 \gamma^{\prime}$ s in the final state. As can be seen, $\gamma^{\prime}$ 's are efficiently detected by the $\mathrm{CMS}^{5}$ electromagnetic calorimeters (BARREL and ENDCAP). But CMS cannot trigger on the protons scattered with small angles $(10 \leq \eta \leq 14)$. This can be made using the TOTEM ${ }^{6}$ forward tracking detectors located inside the beam-pipe, the so-called Roman Pots (RP). TOTEM will install several RP stations at distances from 100 to 215 meters along the beam. The general scheme of the measurements is shown in fig. 6. Using the CMS+TOTEM facilities could be a unique opportunity to study the DPE reactions at LHC.
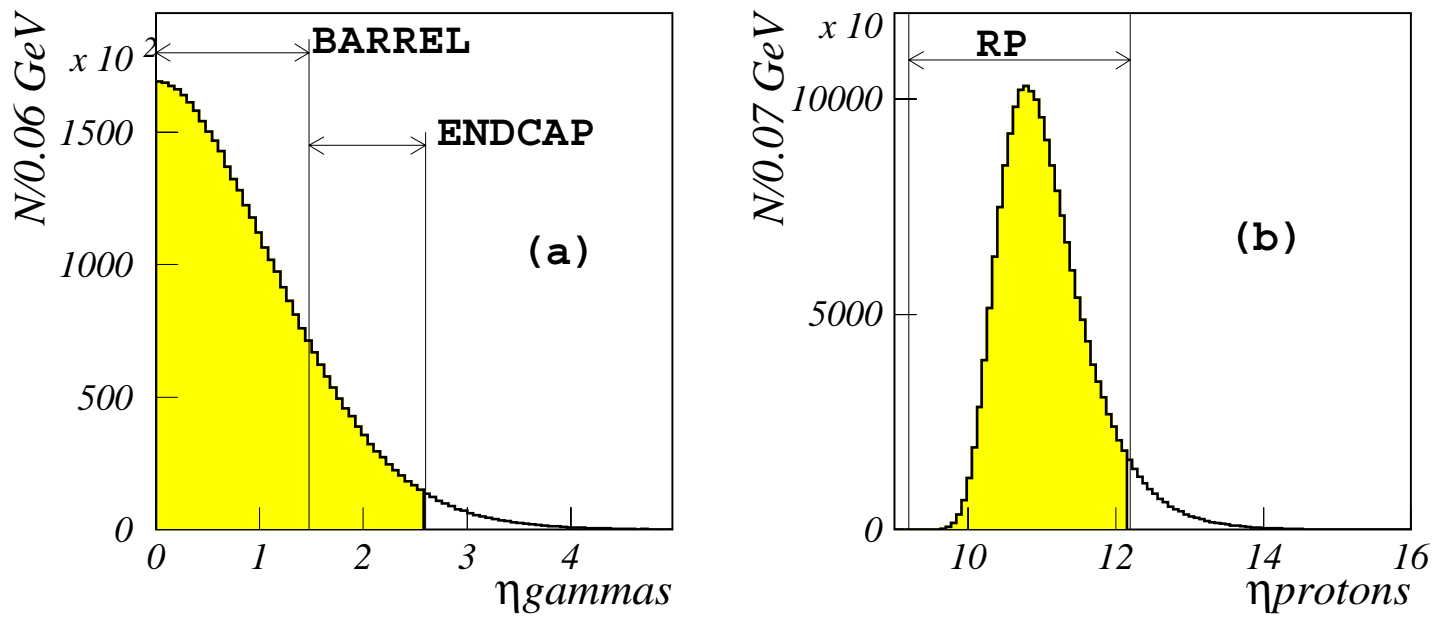

Figure 5: Distributions of pseudorapidity $\eta$ for $\gamma$ 's (a) and protons (b). The $\eta$-sizes of the CMS electromagnetic calorimeters (BARREL and ENDCAP) and of the Roman Pot (RP) are shown.

\footnotetext{
${ }^{5}$ The CMS detector is described in detail in the CMS Technical Proposal [20].

${ }^{6}$ The detailed description of the TOTEM's physical goals, equipments and its integration into CMS can be found in [21]
} 


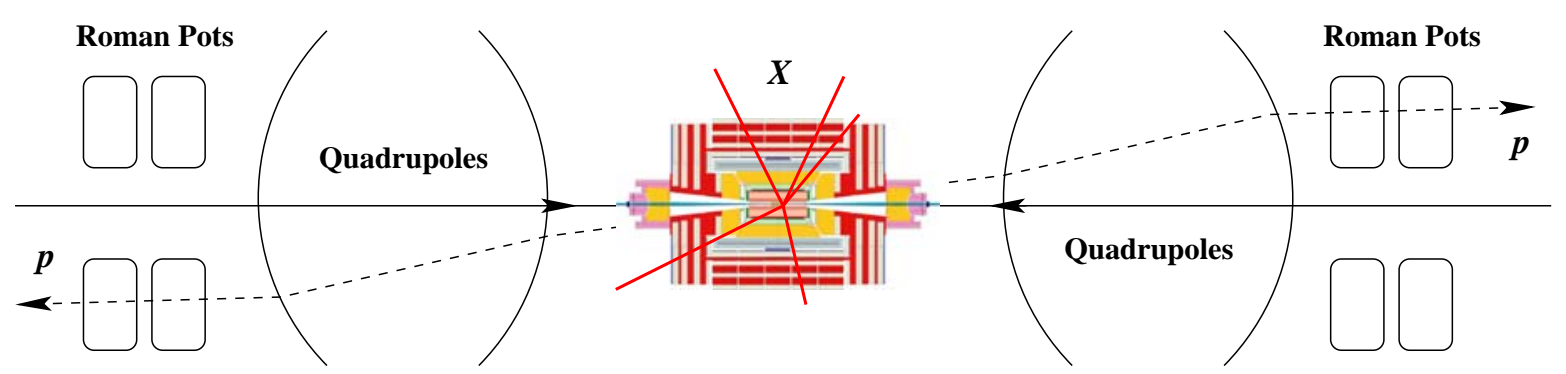

Figure 6: Scheme of the forward protons measurement using Roman pots stations.

To perform successful measurements at very small scattered angles TOTEM needs some special parameters for the beam, which are different from the nominal LHC conditions. It requires to:

- run with high- $\beta$ optics $\left(\beta_{\text {TOTEM }}^{*}=1000 \div 1500 \mathrm{~m}\right)$, instead of the nominal LHC $\beta_{\text {nom }}^{*}=0.5 \mathrm{~m}^{7}$;

- decrease the number of bunches, $n_{\text {TOTEM }} / n_{\text {nom }} \approx 10^{-2}$.

Such conditions led to the decline of the luminosity from $L_{\text {nom }}=10^{34} \mathrm{~cm}^{-2} \mathrm{~s}^{-1}$ to $L_{\text {TOTEM }}=$ $10^{28} \mathrm{~cm}^{-2} \mathrm{~s}^{-1}$. The measurements can be performed in special runs during the early running-in phase of the LHC, taking periods of a few days. As has been mentioned above, the estimated number of DPE events (non-corrected by any efficiency) is about $\approx 350000$ per day at this low luminosity.

To estimate the efficiency of DPE registration, resolution and background conditions, we used the program CMSJET[22] adapted to our purposes. This program provides a fast non-GEANT simulation of the CMS detector response. The reaction 1 with DPE, at $\sqrt{s}=14 \mathrm{TeV}$, has been generated using a modified version of the WA102 event generator. The distribution in $x_{F}$ of outgoing protons and in $t$, the four momentum transfer squared of the proton vertices, measured and parametrized by the WA102 experiment are used in the generation. The $s$ dependence of these variables were taken into account according to reference [11]. The generation was made for neutral and charged decay channels of the central particle $X$. A detailed description of the generator and the results of the simulations are presented in reference [23]. Here we show some important figures and outcomes.

The mass dependence of the efficiency for the reaction $p p \rightarrow p X p, X \rightarrow 2 \pi^{o} \rightarrow 4 \gamma$ is shown in fig.7. The low energy gammas decrease the efficiency essentially because the noise terms in the resolution of the electromagnetic calorimeters are rather large. One can see on this figure that the gamma conversion and the energy cut in the Barrel ECAL strongly suppress the detection of the events. The mass resolution of the calorimeter

\footnotetext{
${ }^{7}$ The $\beta$-function is an optical function of the collider defining the transverse size of the beam $\left(\sigma_{x, y}^{*} \sim\right.$ $\left.\sqrt{\beta^{*}}\right) . \beta^{*}$ is the value of the $\beta$-function at the interaction points.
} 


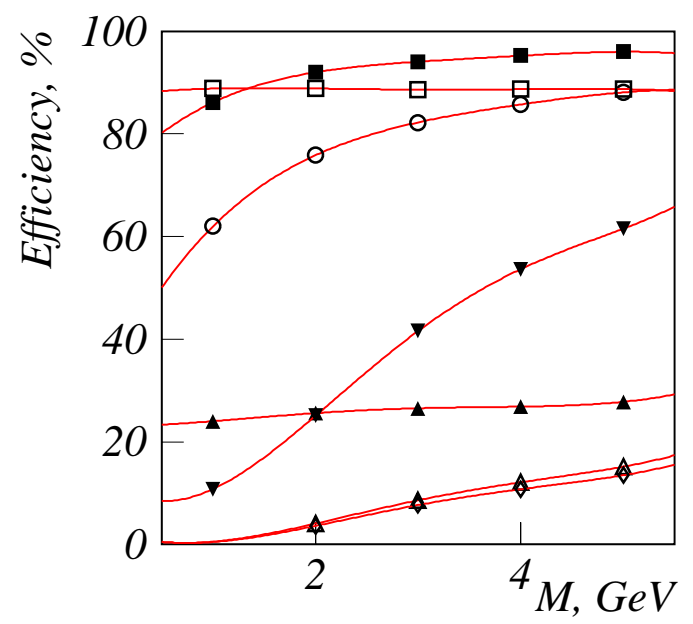

$$
\begin{aligned}
& p p \rightarrow p X p \\
& X \rightarrow \pi^{O} \pi^{O} \rightarrow 4 \gamma \\
& \text { - } 4 \text { gammas in ECAL } \\
& \triangle \text { conversion to } e^{+} e^{-} \\
& \text {energy cut in Barrel } \\
& \text { energy cut in Endcap } \\
& \text { protons efficiency } \\
& \text { gammas efficiency } \\
& \text { total efficiency }
\end{aligned}
$$

Figure 7: Mass dependence of the efficiency for the reaction $p p \rightarrow p X p, X \rightarrow 2 \pi^{o} \rightarrow 4 \gamma$. The curves show the contribution to the total efficiency of the different factors suppressing the registration of events.

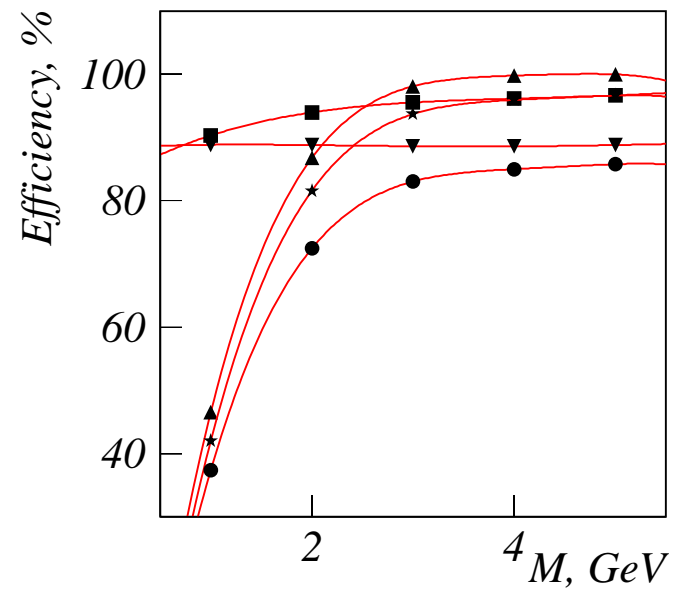

$$
\begin{aligned}
& p p \rightarrow p X p \\
& X \rightarrow \pi^{+} \pi^{-}
\end{aligned}
$$

- 2 tracks in Tracker

- 2 tracks in Calorimeters

- protons efficiency

* tracks efficiency

- total efficiency

Figure 8: Mass dependence of the efficiency for the reaction $p p \rightarrow p X p, X \rightarrow \pi^{-} \pi^{+}$. The curves show the contribution to the total efficiency of the different factors suppressing the registration of events. 
has been studied as a function of the $X$ mass. The relative resolution on the $X$ mass, $d \sigma_{M} / d M$, decreases from $25 \%$ at $1 \mathrm{GeV}$ to $10 \%$ at $5 \mathrm{GeV}$.

The same study has been performed for the charged decay channel of the $X$ particle: $p p \rightarrow p X p, X \rightarrow \pi^{-} \pi^{+}$. It was required that 2 charged pions should be detected by the CMS tracker and the 2 protons by the Roman Pots. The result for the efficiency is shown in fig.8.

The efficiency for tracks detection increases rapidly and becomes higher than the efficiency for proton detection above $2.5 \mathrm{GeV}$. Thus, above $3 \mathrm{GeV}$ the efficiency is very high (close to 90\%) and is only limited by the efficiency for proton detection. The mass resolution is about $0.7 \%$ above $2 \mathrm{GeV}$ and rises up to $\approx 1.2 \%$ at $1 \mathrm{GeV}$, which is much better than in the case of neutral decays.

In order to investigate the background to the central production, we use the standard CMS set of PYTHIA's parameters for minimum bias ${ }^{8}$. In addition the processes which have similar kinematics to double Pomeron exchange (DPE), such as elastic scattering, single diffraction, double diffraction, are included in the background. All processes, generated for the background study, are listed in table 2. We included the process of DPE to PYTHIA [24], using the above mentioned generator, and numbered it as 200. We suggest

\begin{tabular}{|l|c|c|}
\hline Process & $\begin{array}{c}\text { Number in } \\
\text { PYTHIA }\end{array}$ & $\begin{array}{c}\text { Cross-section, } \\
\text { mb }\end{array}$ \\
\hline$f f^{\prime} \rightarrow f f^{\prime}(\mathrm{QCD})$ & 11 & 1.0880 \\
$f \bar{f} \rightarrow f^{\prime} \bar{f}^{\prime}$ & 12 & 0.0193 \\
$f \bar{f} \rightarrow g g$ & 13 & 0.0142 \\
$f g \rightarrow f g$ & 28 & 15.1700 \\
$g g \rightarrow f \bar{f}$ & 53 & 1.0800 \\
$g g \rightarrow g g$ & 68 & 37.8500 \\
Elastic scattering $p p \rightarrow p p$ & 91 & 22.2100 \\
Single diffractive $p p \rightarrow X p$ & 92 & 7.1513 \\
Single diffractive $p p \rightarrow p X$ & 93 & 7.1513 \\
Double diffractive $p p \rightarrow X_{1} X_{2}$ & 94 & 9.7800 \\
Low- $p_{T}$ scattering & 95 & 0.0002 \\
DPE $p p \rightarrow p X p$ & 200 & 0.3700 \\
All included processes & & 101.4000 \\
\hline
\end{tabular}

Table 2: Processes generated for the background study.

some requirements for the DPE selection which can be used for the trigger or for the off-line event analysis. The main one is the detection of one track in the forward Roman pots and one track in the backward ones. We use also different energy deposits in the

\footnotetext{
${ }^{8}$ http://cmsdoc.cern.ch/cms/Physics/btau/www/MB_cards.html
} 
CMS calorimeters for the background and for the DPE events and the absence of tracks in the TOTEM inelastic tracking detectors T1 and T2 covering a pseudorapidity region from 3 to 7 . Such selection requirements allow a very effective background suppression, saving more than $85 \%$ of the DPE events. The ratio of DPE to background before and after selections is the following:

$$
\left(\frac{N_{D P E}}{N_{b g}}\right)_{\text {before selection }} \approx 0.004, \quad\left(\frac{N_{D P E}}{N_{b g}}\right)_{\text {after selection }} \approx 20 .
$$

We can conclude that the CMS+TOTEM facility gives a unique and real opportunity to study reaction 1 at the LHC energy in the region of small mass $(<10 \mathrm{GeV})$ of the central system $X$, where an intensive production by DPE of gluon rich states is predicted.

\subsection{High luminosity physics}

Measurements of the high mass central systems in reaction 1, for example Higgs or Radion, which have an extremely small cross-section are impossible at the luminosity $L_{\text {TOTEM }}=10^{28} \mathrm{~cm}^{-2} \mathrm{~s}^{-1}$. But they can be made at the nominal LHC luminosity $10^{34}$. Taking into account the most pessimistic estimates of the cross-section for the central Higgs production, $3 \mathrm{fb}$, one expects 600 events per year (without taking in account any efficiency). The increase of the luminosity should be offset by a decrease in $\beta^{*}\left(\beta_{\text {nom }}^{*}=\right.$ $0.5 \mathrm{~m}$ ) and, as a consequence, the worsening of some beam parameters, as the beam momentum and angular spread. The transverse size of the beam becomes larger at the distances $100 \div 200$ meters, where TOTEM's RPs are located, and one can not use them for measurements of the diffractive scattered protons flying inside the beam. For the high luminosity runs it is suggested to install several additional RP stations at distances $300 \div 450$ meters. One of the possible setup is shown in fig.9.

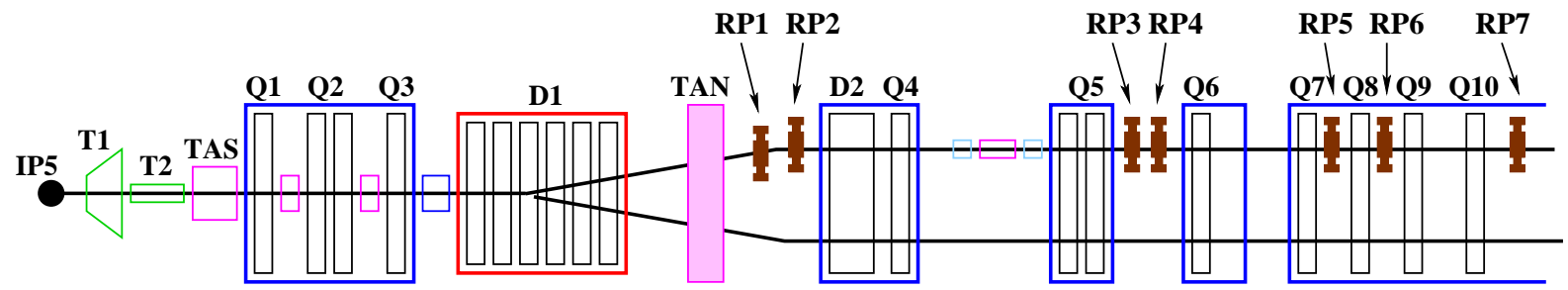

Figure 9: LHC beam line with the 7 RPs. Here, IP5 is the CMS interaction point, T1 and $\mathrm{T} 2$ are inelastic tracking detectors of TOTEM, TAS and TAN are collimators, $\mathrm{D}_{i}$ and $\mathrm{Q}_{i}$ are dipole and quadrupole magnets of $\mathrm{LHC} \mathrm{RP}_{i}$ are Roman Pot stations of TOTEM.

For the optimization of the RPs positions and for the calculations of the efficiency and resolution of the forward protons detection by the tracking detectors inside RPs a special interface program has been created to link PYTHIA [24] and MAD [25] (Methodical 
Accelerator Design, that tracks protons in the LHC). The block-scheme of the program is shown in fig.10. The program allows to calculate the trajectories of the protons scattered in the different physical processes generated by PYTHIA (or any program compatible with it) and then traced inside the beam-pipe. One bank of data carries the coordinates and momenta of the protons, as scattered as beam, at any points along the beam that one needs to study. The beam envelope, the shape of the scattered protons current can easily be plotted at different projections, transversal or longitudinal, at any point along the beam, see fig.11. The spread of the beam momentum, the spread of the production angle and of the coordinates of the interaction at the interaction point (IP) can be included in the simulations.

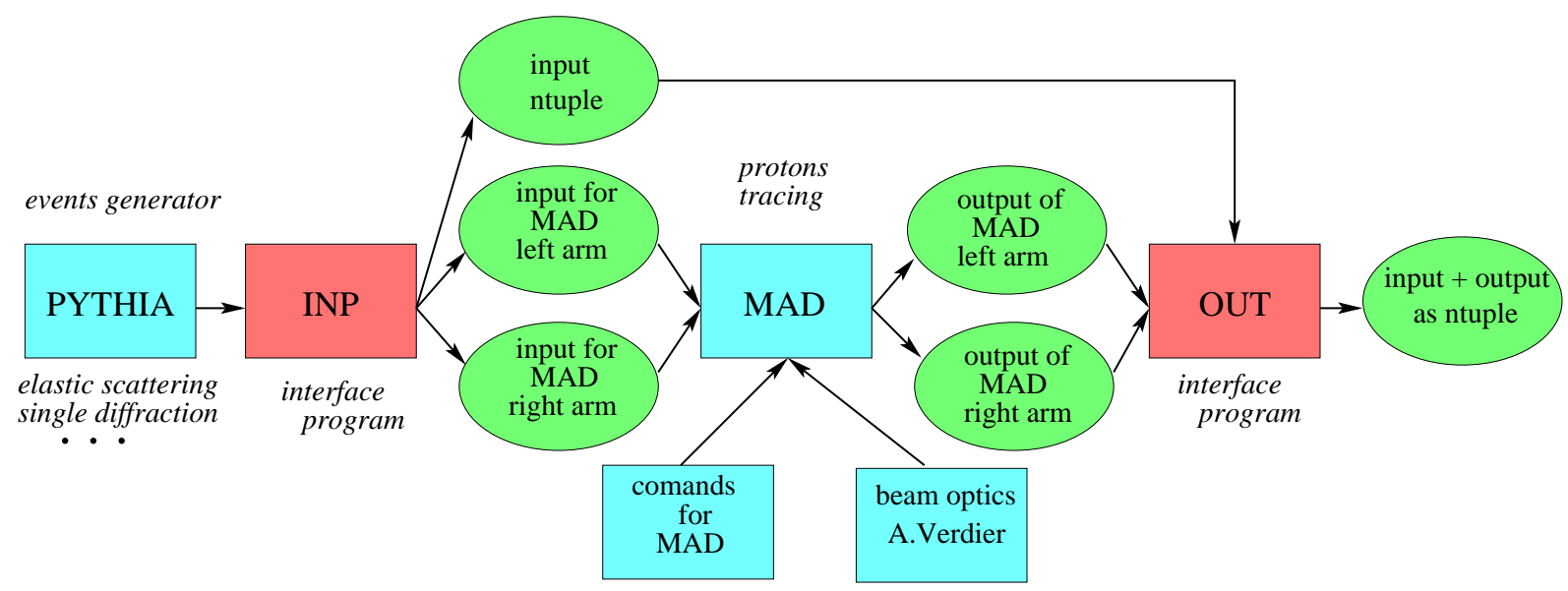

Figure 10: The scheme of the interface program to link the programs PYTHIA and MAD.

The trajectory of the proton (as scattered as beam) is defined exclusively by 6 parameters at the IP. One can choose, for example, the following set: $\left(x^{*}, y^{*}, \theta_{x}^{*}, \theta_{y}^{*}, \xi\right)$, where $\left(x^{*}, y^{*}\right)$ are the transverse coordinates at the IP, $\left(\theta_{x}^{*}, \theta_{y}^{*}\right)$ are the production angles, $\xi=\frac{\delta p}{p}$ is the relative momentum loss of the proton. If one measures the $\left(x_{i}, y_{i}\right)$ coordinates of the proton by the RPs tracking detectors at different points $z_{i}$ along the beam and if one knows all trajectories for different initial parameters with MAD one can reconstruct the parameters of the proton at the interaction point. The most important of them, defining the diffractive process, are $t=p^{2}\left(\theta_{x}^{* 2}+\theta_{y}^{* 2}\right)$, the transverse momentum squared, and $\xi$. $\xi$ is very important and should be measured for reaction 1 , because if one measures $\xi_{1}$ for the forward proton and $\xi_{2}$ for the backward proton one can calculate the mass of the central system $X$ by the simple formula (Missing Mass Method):

$$
M_{X}^{2}=s \xi_{1} \xi_{2}
$$

So one can calculate the $X$ mass using the measurements of the RPs only, without $\mathrm{CMS}^{9}$.

\footnotetext{
${ }^{9}$ But one needs the CMS information at least for the trigger.
} 

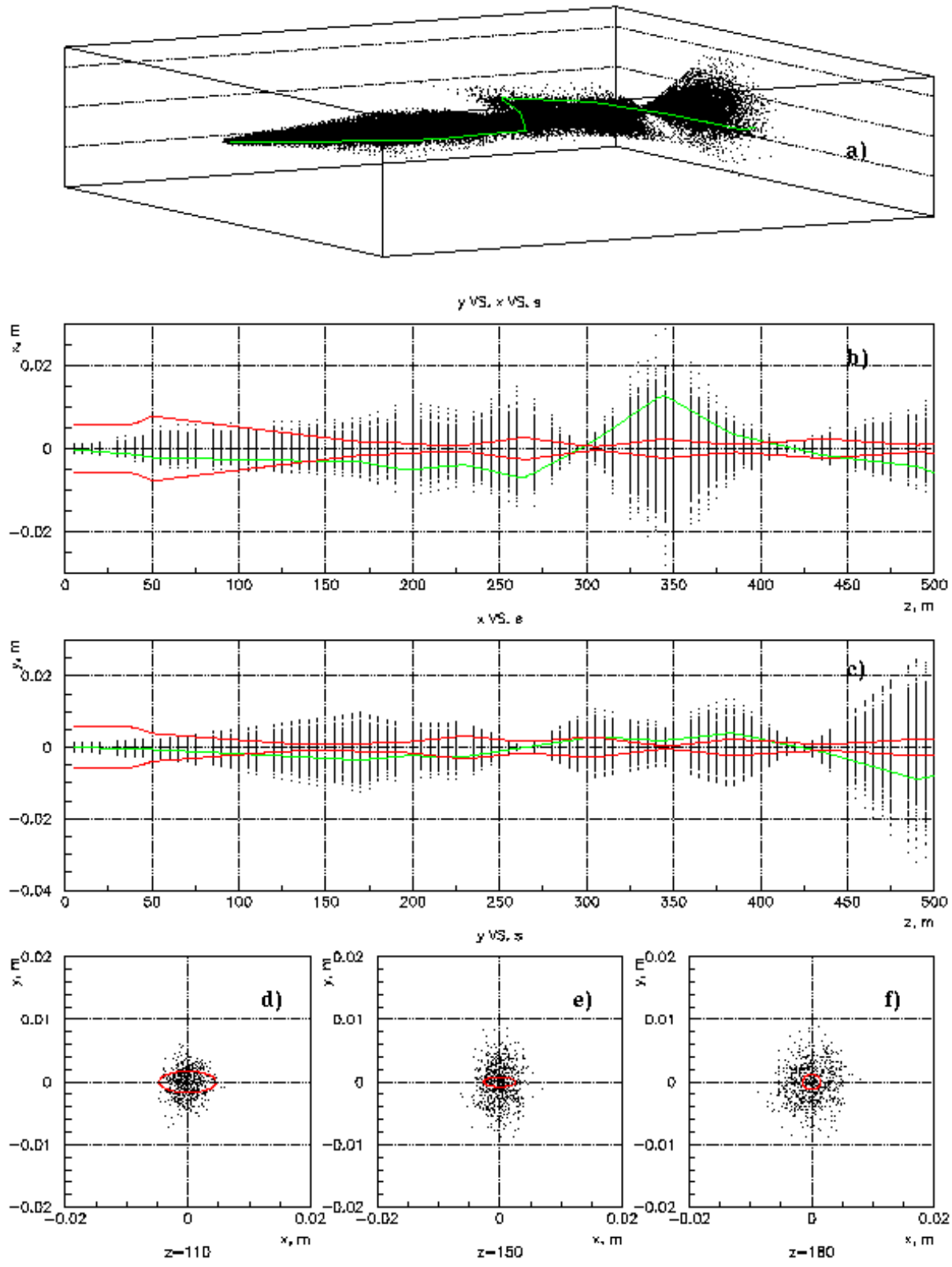

Figure 11: Elastic scattered protons $\left(\beta^{*}=1100 \mathrm{~m}\right)$. a) 3D view of the proton current; b) horizontal projections of a) ; c) vertical projections of a); d) transverse projections of a) at different distances along the beam $(z=110,150$ and $180 \mathrm{~m})$. The red lines show an envelope at $15 \sigma$ of the beam, the green line shows a trajectory of one beam proton. 
We made an estimate of the efficiency for proton detection by RPs located as shown in fig.9 and of the $\xi$ resolution assuming a $30 \mu \mathrm{m}$ coordinate resolution for the RPs tracking detectors. The calculations were made for an LHC optic related to $\beta^{*}=0.5 \mathrm{~m}$. The result is shown in fig.12. The green area on the $(z, \xi) 2 \mathrm{D}$ plot shows the region of a non-zero efficiency for the proton detection. One can see that RP 5, 6 and 7 cover together the $\xi$ interval from 0.002 to 0.02 . Using equation 3 one can calculate the interval of the central masses which is measured by the missing mass method: from 28 to $280 \mathrm{GeV}$. This interval widely covers the limits on the Higgs mass obtained by LEPII and by a global fit of the Standard Model parameters: $114 \mathrm{GeV}<M_{H}<205 \mathrm{GeV}$ [26]. The relative $\xi$ resolution and, accordingly, mass resolution in this interval vary from 2 to $5 \%$ depending on the $\xi$ value, as can be seen in fig.12. This is a first rough estimate which can be improved, but it is already promising for such a method. This has to be compared with the most optimistic estimate of the Higgs mass resolution via the $H \rightarrow \gamma \gamma$ measurement that gives $1 \%$.

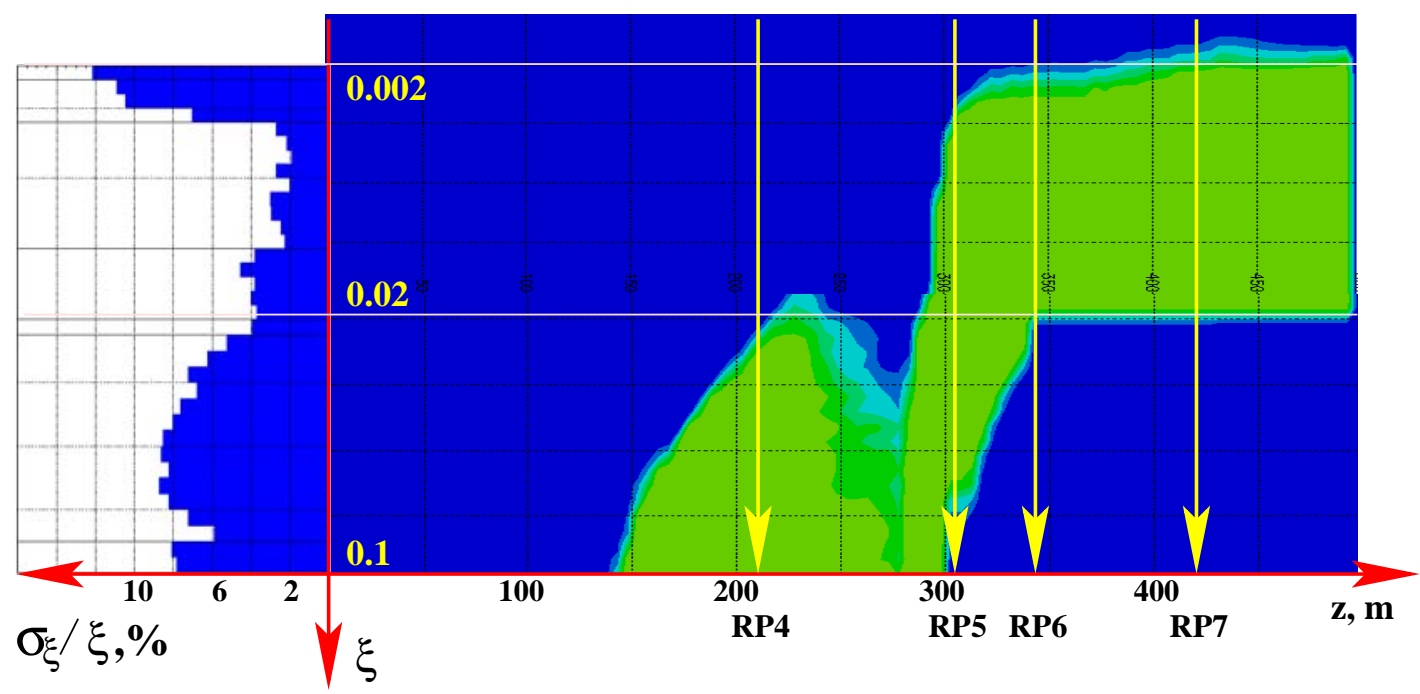

Figure 12: The right 2D histogram shows the efficiency for forward proton detection as a function of $\xi$, the relative proton momentum loss, and $z$, the distance along the beam. The RPs positions are shown by arrows. The left histogram presents relative resolution of the $\xi$ measurements by RPs 4, 5, 6 and 7 .

At present there is no full GEANT-like simulation for the background to the Higgs production (reaction 1), but analytical estimates were made in the reference [18] for the exclusive Higgs production $p p \rightarrow p H p, H \rightarrow b \bar{b}$. As has been mentioned above, the authors of [18] estimate the significance $S / \sqrt{S+B}$ of the Higgs observation during one year LHC run at a luminosity $10^{33}$ to be about $3 \sigma$ even for the more pessimistic $(3 \mathrm{fb})$ Higgs cross-section. It makes the reaction 1 competitive to the other methods of Higgs 
measurements (for example, for the channel $H \rightarrow \gamma \gamma: S / \sqrt{S+B} \approx 4 \sigma$ ).

Recently, new theoretical works have been published devoted to the calculation of the azimuthal angle between protons in the final state of reaction 1 as was done in the model of F.Close [3]. In [27] the authors try to estimate the behaviour of this angle for the SM scalar and pseudoscalar Higgses produced by DPE in reaction 1. If one knows from the theory such interdependence between $J^{P C}$ of the central particle and the above-mentioned azimuthal angle $\phi_{p p}$ one can solve the inverse problem: defining the quantum numbers $J^{P C}$ of the central particle by measuring $\phi_{p p}$. Contrary to the traditional determination of $J^{P C}$ of the particle by the analysis of the angular distributions of its decay products (partial-wave analysis) one can define these quantum numbers by measuring the forward protons only, making the procedure much simpler.

\section{Conclusion}

We conclude that the CMS+TOTEM facility gives a unique opportunity to study the double Pomeron exchange at the LHC. Low mass central systems, having a high crosssection production in DPE (about $0.37 \mathrm{mb}$ ) can be studied at low luminosity $\left(L_{\text {TOTEM }}=\right.$ $10^{28} \mathrm{~cm}^{-2} \mathrm{~s}^{-1}$ ) in CMS and TOTEM common runs in the early running-in phase of the LHC. High mass central objects, like Higgs produced diffractively in DPE, have smaller cross-section (a few fb) and can be studied at the nominal LHC luminosity $\left(L_{n o m}=\right.$ $\left.10^{34} \mathrm{~cm}^{-2} \mathrm{~s}^{-1}\right)$. Such study needs additional Roman Pots tracking detectors located at distances 300-400 $\mathrm{m}$ along the beam on both sides of CMS.

The study of the central production reaction at the LHC energy, where a pure double Pomeron exchange is predicted, gives a feeling of great achievement to understand the nature of the Pomeron and the underlying dynamics of the double Pomeron exchange, to solve the "glueball puzzle" and it gives a unique opportunity to make measurements of the exclusive Higgs production in DPE at LHC.

In this paper, CMS is given as an example but is also valid for ATLAS. In this context, BARREL and ENDCAP (two sub-detectors of CMS) should be replaced by their equivalent sub-detectors of ATLAS (with their pseudorapidity limits). In this way, TOTEM (an experiment with Roman pots in the beam pipe) should be understood, in the case of ATLAS, as Roman pots in the beam pipe on both sides of ATLAS.

\section{Acknowledgements}

We would like to thank Professor V.A. Petrov and R.A. Ryutin (IHEP) for their valuable assistance. One of us, Andrei Sobol, thanks IN2P3 for having offered a foreign visitor position at LAPP. 


\section{References}

[1] D.Barberis et.al. Phys. Lett. B422 (1998) 399, Phys. Lett. B427 (1998) 398, Phys. Lett. B432 (1998) 436, Phys. Lett. B436 (1998) 204, Phys. Lett. B440 (1998) 225, Phys. Lett. B446 (1999) 342, Phys. Lett. B453 (1999) 305, Phys. Lett. B453 (1999) 316, Phys. Lett. B453 (1999) 325, Phys. Lett. B462 (1999) 462, Phys. Lett. B467 (1999) 165, Phys. Lett. B471 (2000) 429, Phys. Lett. B471 (2000) 435, Phys. Lett. B471 (2000) 440, Phys. Lett. B474 (2000) 423, Phys. Lett. B479 (2000) 59, Phys. Lett. B484 (2000) 198, Phys. Lett. B485 (2000) 357, Phys. Lett. B488 (2000) 225, Phys. Lett. B507 (2001) 14.

[2] F.E.Close Phys. Lett. B419 (1998) 387;

P.Castoldi et.al. Phys. Lett. B425 (1998) 359;

N.I.Kochelev, hep-ph/9902203;

N.I.Kochelev et.al. Phys. Lett. B457 (1999) 202;

F.E.Close and G.Schuler Phys. Lett. B458 (1999) 127;

E.Shuryak and I.Zahed Phys.Rev. D68:034001 (2003).

[3] F.E.Close and G.Schuler Phys. Lett. B464 (1999) 279.

[4] F.E.Close, A.Kirk and G.Schuler Phys. Lett. B477 (2000) 13.

[5] F.E.Close and A.Kirk Phys. Lett. B483 (2000) 345.

[6] C.Amsler and F.E.Close Phys. Lett. B353 (1995) 385;

W.Lee and D.Weingarten, Phys.Rev. D61 (2000) 014015;

D.Li, H.Yu and Q.Shen hep-ph/0001107;

L.S.Celenza et.al. Phys.Rev. C61 (2000) 035201;

M.Strohmeier-Presicek et.al. Phys.Rev. D60 (1999) 054010.

[7] E.Klempt "Meson Spectroscopy: Glueballs, Hybrids and $Q \bar{Q}$ Mesons", PSI Zuoz Summer School, August 13-19, 2000; hep-ex/0101031.

[8] A.Brandt et.al. Eur.Phys.J.C. 25 (2002) 361; hep-ex/0205037.

[9] S.N.Ganguli and D.P.Roy Phys.Rep. 67 (1980) 203.

[10] A.Kirk Phys. Lett. B489 (2000) 29.

[11] G.Schuler and T.Sjöstrand Phys.Rev. D49 (1994) 2257-2267.

[12] A. Bialas and P.V. Landshoff Phys.Lett.B256 (1991) 540.

[13] J.R.Cudell, O.F.Hernandez Nucl.Phys B471 (1996) 471; hep-ph/9511252. 
[14] D.E.Kharzeev and E.Levin Phys.Rev. D63 (2001) 073004; hep-ph/0005311.

[15] V.Petrov and R.Ryutin Talk on the CMS-TOTEM diffraction meeting, 8 March, 2002; http://totem.web.cern.ch/Totem/cms/meeting.html

[16] V.A.Khoze, A.D.Martin and M.G.Ryskin Eur.Phys.J.C23 (2002) 311; hep-ph/0111078.

[17] V.Petrov and R.Ryutin "Exclusive Double Diffraction Higgs Boson Production at $L H C "$, 2003, to be published in Phys.Lett.

[18] A.De Roeck, V.A.Khoze, A.D.Martin and M.G.Ryskin Eur.Phys.J. C25 (2002) 391; hep-ph/0207042.

[19] V.Petrov Private communications, 2002-2003 yy.

[20] CMS Technical Proposal, CERN/LHCC 94-38, December 1994.

[21] TOTEM Technical Proposal, CERN/LHCC 99-7, March 1999.

[22] S.Abdullin et.al. CMS TN/1994-180.

[23] A.Sobol "A study of the centrally produced $\eta \pi^{o}$ and $\eta \pi^{-}$systems in $p p$ interactions". Doctor thesis, Protvino-Annecy-les-Vieux, 2001; http://wwwlapp.in2p3.fr

[24] T.Sjöstrand Computer Physics Commun. 82 (1994) 74.

[25] H.Grote et.al. The MAD Program, Version 8.19, CERN/SL/90-13, 1996 y.

[26] M.S.Chanowitz LBNL-52452, Feb 2003; hep-ph/0304199.

[27] A.B.Kaidalov, V.A.Khoze, A.D.Martin and M.G.Ryskin IPPP-03-35, DCPT-03-70, Jul 2003; hep-ph/0307064. 